\title{
The Impact of Nutrients on Mental Health and Well-Being: Insights From the Literature
}

\author{
Maurizio Muscaritoli* \\ Department of Translational and Precision Medicine, Sapienza University of Rome, Rome, Italy
}

A good nutritional status is important for maintaining normal body function and preventing or mitigating the dysfunction induced by internal or external factors. Nutritional deficiencies often result in impaired function, and, conversely, intakes at recommended levels can resume or further enhance body functions. An increasing number of studies are revealing that diet and nutrition are critical not only for physiology and body composition, but also have significant effects on mood and mental well-being. In particular, Western dietary habits have been the object of several research studies focusing on the relationship between nutrition and mental health. This review aims to summarize the current knowledge about the relationship between the intake of specific

OPEN ACCESS

Edited by:

David Vauzour,

University of East Anglia,

United Kingdom

Reviewed by:

Eva Ntanasi,

National and Kapodistrian University

of Athens, Greece

Yannick Vermeiren,

Wageningen University and

Research, Netherlands

${ }^{*}$ Correspondence:

Maurizio Muscaritoli

maurizio.muscaritoli@uniroma1.it

Specialty section:

This article was submitted to

Nutrition and Brain Health,

a section of the journal

Frontiers in Nutrition

Received: 20 January 2021

Accepted: 18 February 2021

Published: 08 March 2021

Citation:

Muscaritoli M (2021) The Impact of

Nutrients on Mental Health and

Well-Being: Insights From the

Literature. Front. Nutr. 8:656290.

doi: 10.3389/fnut.2021.656290 micro- and macronutrients, including eicosapentaenoic acid, docosahexaenoic acid, alpha-tocopherol, magnesium and folic acid, and mental health, with particular reference to their beneficial effect on stress, sleep disorders, anxiety, mild cognitive impairment, as well as on neuropsychiatric disorders, all significantly affecting the quality of life of an increasing number of people. Overall data support a positive role for the nutrients mentioned above in the preservation of normal brain function and mental well-being, also through the control of neuroinflammation, and encourage their integration in a well-balanced and varied diet, accompanied by a healthy lifestyle. This strategy is of particular importance when considering the global human aging and that the brain suffers significantly from the life-long impact of stress factors.

Keywords: eicosapentaenoic acid, docosahexaenoic acid, alpha-tocopherol, magnesium, folic acid, mental health, well-being, inflammation

\section{INTRODUCTION}

A healthy dietary pattern can affect mental health and well-being through anti-inflammatory, antioxidant, neurogenesis, microbiome- and immune-modifying mechanisms, as well as through epigenetic modifications (1). Dietary profile affects not only the brain composition, structure and function, but also endogenous hormones, neuropeptides, neurotransmitters, and the microbiotagut-brain axis, in turn playing a key role in modulation of stress and inflammation and in preservation of cognitive function (2). In addition to a healthy and balanced diet, the supplementation of micronutrients (e.g., vitamins and minerals) and macronutrients (e.g., fatty acids) can provide several beneficial effects, due to their multiple biological roles (3). This narrative review aims to summarize the current knowledge about the relationship between the intake of specific nutrients, including the omega-3 polyunsaturated fatty acids (PUFAs) eicosapentaenoic acid (EPA) and docosahexaenoic acid (DHA), alpha-tocopherol, magnesium and folic acid, and 
the beneficial effect on mental health and well-being (Figure 1). A comprehensive search and critical review have been conducted in PubMed database using the keywords stress, anxiety, sleep disorders, mild cognitive impairment (MCI), depression, bipolar disorders, obsessive-compulsive disorder, neuroinflammation, inflammation, associated with the nutrients alpha-tocopherol, folic acid/folate, magnesium, omega-3 fatty acids, EPA, and DHA through the use of the Boolean operators AND, OR, identifying the articles relevant to this review. The literature search has been limited to English language articles. Additionally, manual searches for related articles have also been performed. The time range for literature search has been 1994-2020, with about 70\% of articles published in 2016-2020. Randomized clinical trials (RCTs) have been prioritized in the selection of research studies to be reviewed; however, some pre-clinical studies in animal models of disease have been discussed as well.

EPA and DHA are the predominant PUFAs, are endowed with anti-inflammatory properties and exert various biological activities, mainly as substrates for the biosynthesis of specialized pro-resolving mediators (SPMs) and as agonists of cellular receptors (4). DHA is highly concentrated in the central nervous system (CNS), where it plays a key role in optimal development and later cognitive functioning (5). Conversely, steady-state concentrations of EPA are low in the brain, due to its rapid metabolism (6). DHA decreases in the brain during aging and this should be of particular concern in Western countries, where the diet might be poor of omega-3 PUFAs (7). Of interest, EPA and DHA can be converted in their respective endocannabinoid derivatives, eicosapentaenoyl-ethanolamine (EPEA), and docosahexaenoyl-ethanolamine (DHEA), with, among others, potential antidepressive effects (8).

Alpha-tocopherol is one of the eight isoforms of vitamin E, located mainly in cell membranes, where it increases the regularity of lipid packaging. Alpha-tocopherol exerts a protective antioxidant and anti-inflammatory activity that efficiently prevents lipid peroxidation. It accumulates particularly at sites where free radical production is higher, such as the mitochondrial membranes. Alpha-tocopherol is essential for neurological development (9) and is one of the most effective nutrients known to modulate the immune function. A deficiency of vitamin E can occur in the setting of chronic fat malabsorption or lipoprotein deficiency, or derive from low dietary amounts, more frequently in children than in adults. Alpha-tocopherol deficiency impairs both the innate and the adaptive immune function (10), can increase adverse events during pregnancy and lead to neurological abnormalities or disorders (11).

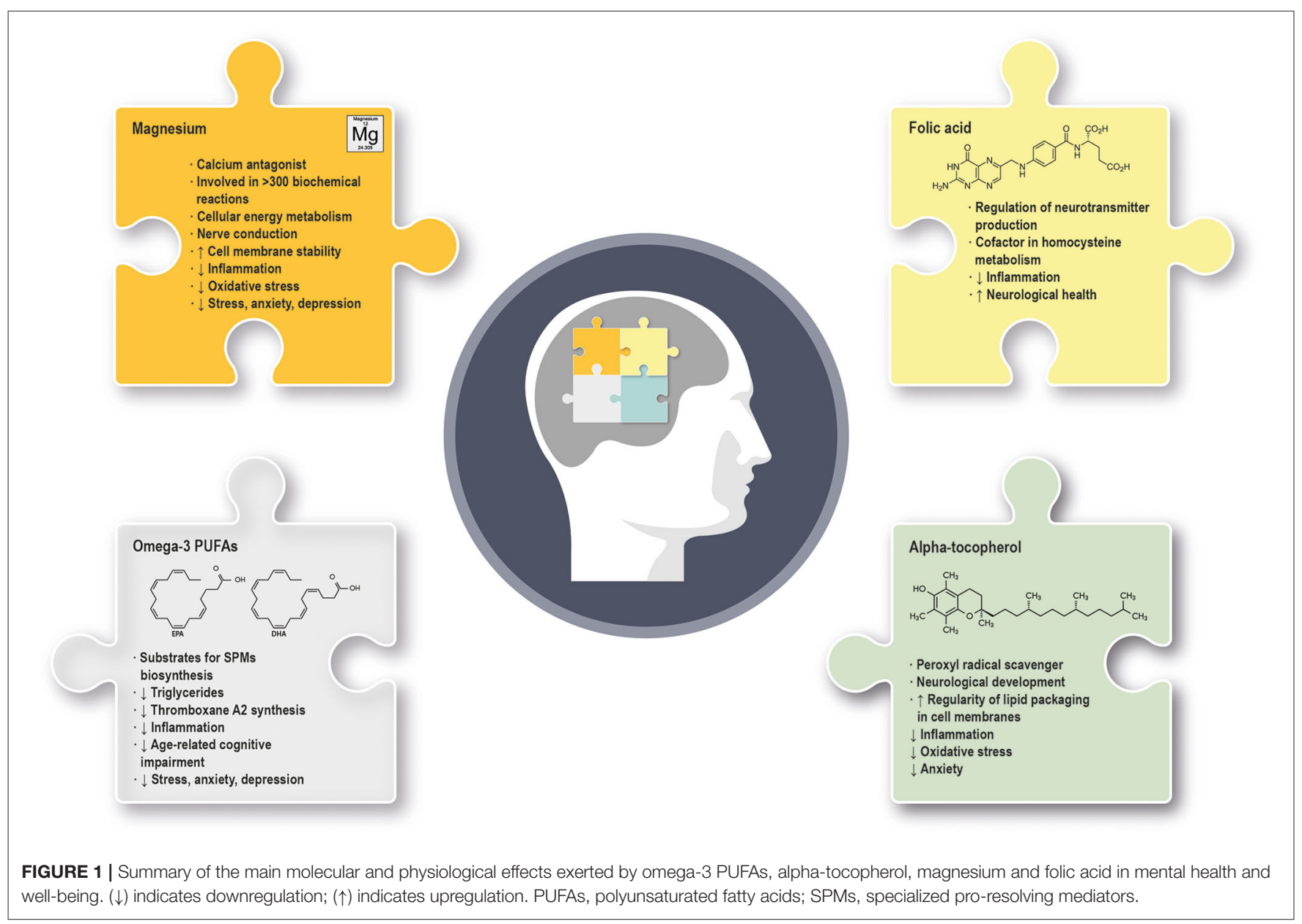


Folic acid is the synthetic form of the water-soluble vitamin B9, also known as folate. Both folate and folic acid must be metabolized into the more bioavailable form L-methylfolate to play their several roles. L-methylfolate is able to cross the blood-brain barrier $(\mathrm{BBB})$ and regulate the production of the neurotransmitters dopamine, norepinephrine and serotonin, thus contributing to mental function and performance (12). Several B-vitamins, including the B9, are also cofactors in homocysteine metabolism and are able to reduce inflammation caused by high levels of this amino acid (13). Folic acid deficiency is caused mainly by inadequate dietary intake and can lead to neurological symptoms in adults and to neural tube defects in the fetus (3).

Magnesium is the second most abundant intracellular cation in human body, acts as calcium antagonist and has a wide range of biological roles, including cellular energy metabolism, nerve conduction, membrane stability, and synaptic transmission. Magnesium has also anti-inflammatory properties mediated by the reduced expression and release of pro-inflammatory molecules (14). Hypomagnesemia usually occurs in critically ill patients due to gastrointestinal loss or dysregulation of renal reabsorption. Also, certain drugs and alcohol use can cause hypomagnesemia (3). Of note, hypomagnesemia can be frequently due to the soil used for agriculture and to extensive intake of processed food and refined grains (15).

\section{NUTRITION AND MENTAL HEALTH PRESERVATION OR RESTORATION: CAN OMEGA-3 FATTY ACIDS, ALPHA- TOCOPHEROL, MAGNESIUM AND FOLIC ACID AFFECT STRESS, ANXIETY, SLEEP DISORDERS AND MILD COGNITIVE IMPAIRMENT?}

\section{Potential Effects on Stress}

Stress is a body reaction to the disturbance of a status of equilibrium. Stress contributes to an overall reduction of the quality of life (QoL) and to a wide range of disorders, including hypertension, cardiovascular disease, inflammatory bowel syndrome, diabetes mellitus (16). In particular, stress can lead to a prolonged release of cortisol and subsequent activation of immune cells, especially neutrophils, followed by the production of free radicals $(17,18)$. Nutritional imbalance, insufficient vitamin intake, and excessive consumption of fat can result in dysregulation of stress hormones and inflammation (16). Conversely, a healthy nutrition can greatly contribute to bridge over the stress and reduce the probability of stressrelated disease. In this setting, a RCT supported a positive impact of folate and magnesium oxide supplementation on pro-inflammatory cytokine profiles in subjects experiencing stress, but without significant changes of psychological measures (19). Magnesium supplementation alleviated stress in healthy adults with hypomagnesemia in a phase IV RCT (20). The administration of DHA to stressed healthy subjects in a RCT resulted in a significant reduction of perceived stress, supporting the protective or "adaptogenic" role of omega-3 PUFAs (21). Omega-3 PUFAs also prevented cortisol increase and reduced the symptoms of occupational burnout in a double-blind RCT (22) (Table 1). Significant evidences for an association between alpha-tocopherol levels and stress are still lacking in humans.

\section{Potential Effects on Anxiety}

Anxiety disorders are the most prevalent mental disorders, and produce fear, worry, and a constant feeling of being overwhelmed; they can be triggered by stress conditions and include, among others, panic disorder, agoraphobia, and social anxiety disorder, with a severe impact on the QoL. Several anxiolytic medications, mainly targeting monoamine neurotransmission, have been introduced in the clinical practice for the treatment of anxiety. However, patients may either do not respond to these medications or respond inadequately. Preclinical and clinical studies have shown that supplementation of essential elements can enhance the drugs' anxiolytic effects. The NEURAPRO trial indicated that the administration of omega-3 PUFAs in addition to cognitive behavioral case management could exert therapeutic effects in individuals at ultra-high risk for psychotic disorders with a significant reduction of mood and anxiety disorders (23). These data are consistent with the RCT results obtained by Robinson DG et al. (24), suggesting that an EPA/DHA adjuvant treatment is a potential option for depression and anxiety symptoms in subjects with recent onset psychosis and treated with risperidone (Table 1). Magnesium antagonizes the $\mathrm{N}$ methyl-d-aspartate (NMDA) receptor and exhibits gammaaminobutyric acid receptor agonist activity; these mechanisms may be at the basis of its anxiolytic activity observed in animal models (30). Consistently, a magnesium-deficient diet contributes to enhancement of anxiety-like behavior in murine models (31), while a systematic review suggests a beneficial effect of magnesium on subjective anxiety, but also recommends additional randomized controlled trials to confirm this observation further (32). Vitamin E deficiency during pregnancy in murine models leads to irreversible alterations in brain glutamate levels and is associated with increased anxiety at adulthood, suggesting the importance of vitamin E during pregnancy to prevent increased anxiety in later life (33).

\section{Potential Effects on Sleep Disorders}

Sleep disorders include, among others, insomnia and obstructive sleep apnea, and cause a disruption of the normal circadian rhythm with a negative impact on physical health and mental well-being. It is well-known that dietary components can directly affect sleep quality and duration (34); hence, a proper modulation of nutrients could positively affect sleep disorders. Some pre-clinical studies showed that omega-3 PUFA deficiency affects the sleep/wake patterns in basal condition and after a peripheral immune challenge, possibly through the dysregulation of cytokine release in cortical neuronal networks (35). A RCT enrolling patients affected by major depressive disorder (MDD) revealed that the intake of adjuvant omega-3 PUFAs in addition to a standard medication improved not only symptoms of depression, but also anxiety and sleep disorders when compared 
TABLE 1 | Summary of the clinical trials we reviewed and providing statistically significant results about the beneficial effect of nutrient supplementation on mental health and well-being.

\begin{tabular}{|c|c|c|}
\hline Tested molecule(s) & Disorder & Study Design \\
\hline $\begin{array}{l}\text { Magnesium oxide, folic acid, } \\
\text { multivitamin supplementation }\end{array}$ & Stress & $\begin{array}{l}\text { 8-week randomized controlled trial on women } \\
\text { aged } 25-45 \text { and experiencing psychological } \\
\text { distress. Post-intervention data collected } 8 \\
\text { weeks after the start of the supplement intake }\end{array}$ \\
\hline Magnesium, vitamin B6 & Stress & $\begin{array}{l}\text { 8-week, Phase IV, randomized, controlled, } \\
\text { investigator-blinded, parallel-group trial on } \\
\text { subjects aged } 18 \text { to } 50 \text {, with moderate to } \\
\text { extremely severe stress and hypomagnesemia at } \\
\text { screening }\end{array}$ \\
\hline DHA & Stress & $\begin{array}{l}\text { 6-week double blind randomized } \\
\text { placebo-controlled clinical trial on academic } \\
\text { personnel, aged } 18-60 \text {, experiencing stress }\end{array}$ \\
\hline DHA, EPA & Burnout & $\begin{array}{l}\text { 8-week double-blind and placebo-controlled } \\
\text { intervention on subjects aged 18-65, with } \\
\text { self-reported signs of workplace-related } \\
\text { exhaustion }\end{array}$ \\
\hline Omega-3 PUFAs & $\begin{array}{l}\text { Psychotic } \\
\text { disorders }\end{array}$ & $\begin{array}{l}\text { 6-month multicenter, double-blind, } \\
\text { placebo-controlled trial on subjects aged } 13-40 \text {, } \\
\text { at ultra-high risk for psychotic disorders }\end{array}$ \\
\hline
\end{tabular}

Number of participants

Main results

Reference

60 subjects randomized in an active group or in a Positive impact of nutrient supplementation on placebo group pro-inflammatory cytokine profiles in the active

264 subjects randomized 1:1 to treatment with Significant reduction of perceived stress vs.
either the magnesium-vitamin B6 combination or baseline; no significant difference between the $\begin{array}{ll}\text { either the magnesium-vitamin B6 combination or } & \text { baseline; no significant } \\ \text { magnesium alone } & \text { two treatment groups }\end{array}$ at ultra-high risk for psychotic disorders

DHA, EPA

Omega-3 PUFAs

DHA

Omega-3+Omega-6

PUFAs, antioxidant vitamins

(alpha-tocopherol,

gamma-tocopherol, vitamin A) DHA, EPA

Depression and 16-week placebo-controlled trial on subjects anxiety aged 15-40, with recent onset schizophrenia-spectrum $(n=46)$ or bipolar $(n=4)$ disorders and current psychotic symptoms, treated with risperidone

Major depressive 12-week double-blind, randomized and placebo disorder controlled clinical trial on subjects aged 18-65, with major depressive disorder, treated with sertraline

Sleep disorder 14-18-week longitudinal, randomized, double-blinded, placebo-controlled trial on pregnant women aged 18-35

Mild cognitive 6-month randomized, double-blind,

impairment placebo-controlled trial on subjects aged $\geq 65$ years, with probable mild cognitive impairment

Major depressive 8-week randomized, double-blind, placebo disorder controlled trial on subjects aged $18-80$, with major depressive disorder and high plasma levels of inflammation biomarkers

DHA, EPA

Antenatal depressive 12-week randomized trial on pregnant women disorder disorder
93 subjects randomized in an active group treated with fish oil $(n=16)$, a control group treated with olive oil $(n=14)$, or in a control group with no treatment $(n=63)$

43 subjects randomized in an active group treated with DHA and EPA $(n=22)$, or in a placebo control group $(n=21)$

304 subjects randomized in an active group treated with n-3 PUFA together with cognitive

behavioral case management

(CBCM) $(n=66)$ or in placebo group treated with group

CBCM only $(n=62)$

50 subjects receiving risperidone randomized 1:1 Substantial decrease in depression-anxiety in an active group treated with adjuvant n-3 symptoms in the n-3 PUFA group vs. the placebo $(n=25)$

Significant reduction of perceived stress in the active group vs. control groups

Significant improvement of burnout scores and decrease of saliva morning cortisol secretion

Increases of the erythrocyte $\mathrm{n}-3$ index and $\mathrm{DH}$ levels are predictive of better symptomatic and functional outcomes at month 6 in the active

50 subjects receiving setraline randomized 1:1 in Substantial improvement of symptoms of an active group treated with adjuvant n-3 PUFAs depression, and of dimensions of anxiety and $(n=25)$ or in a placebo control group $(n=25) \quad$ sleep symptoms in the n-3 PUFA group vs. the placebo group

48 pregnant women randomized in an active group receiving DHA-supplemented functional food $(n=27)$ or in a placebo group $(n=21)$ 46 subjects randomized 1:1 in an active group treated with PUFAs $(n=23)$ or in a placebo group $(n=23)$

196 subjects randomized in an active group treated with DHA $(n=66)$, an active group treated with EPA $(n=65)$ or in a control group treated with placebo $(n=65)$

100 subjects randomized in an active group treated with omega-3 PUFAs $(n=49)$ or in a control group treated with placebo $(n=51)$
Significant improvement of infant sleep organization in the first $48 \mathrm{~h}$ in the DHA group vs. the placebo group

Favorable improvement of functional capacity and cognitive function for the participants receiving the supplementation vs. the placebo group

Significant improvement of depressive symptoms in EPA-treated patients with high plasma levels of inflammation biomarkers

Significant association between increased EPA and estradiol and decrease of depressive symptoms in the active group

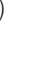

(2)

)

)

)

)

7)


to placebo (25). The intake of a DHA-enriched functional food consumed during pregnancy in a RCT had a beneficial impact on infant sleep organization in the first 48 postnatal hours (26) (Table 1). However, more studies are recommended to further support the positive association between omega-3 PUFA intake and reduction of sleep disorders, as well as to understand the mechanisms by which the modulation of brain omega-3 PUFA levels alters the neuronal circuits underlying sleep/wake states.

\section{Potential Effects on Mild Cognitive Impairment}

MCI has been defined as a transitional stage between healthy aging and dementia, characterized by documented cognitive deficits, together with largely intact daily activities (36). Excessive neuroinflammation triggered by microglia priming associated with aging can affect regions of the brain known to support learning and memory, thus inducing or contributing to MCI. There are currently no approved disease-modifying treatments; consequently, lifestyle modifications, including nutritional habits, have become an important strategy to prevent MCI progression (37). Some nutritional supplements specific for $\mathrm{MCI}$, memory loss and Alzheimer's disease (AD) (e.g., Axona, CerefolinNAC, Souvenaid) are currently marketed and their effectiveness has been analyzed in a variety of meta-analyses, with heterogeneous results (38-40).

An adequate dietary intake of omega-3 PUFAs can slow the age-related MCI and protect against the risk of neurodegenerative dementia (7), and higher levels of plasma EPA+DHA are associated with a lower decline in global cognition and memory (41). In addition, a RCT showed that the supplementation of omega-3+omega-6 PUFAs and antioxidant vitamins, including vitamin $\mathrm{E}$, to older adults affected by $\mathrm{MCI}$ induced an improvement of their condition (27) (Table 1). However, a meta-analysis of RCTs indicated the need of longerterm studies to confirm these effects (42). Of interest, a recent RCT including subjects without cognitive impairment but at risk of dementia, treated with $2,152 \mathrm{mg}$ per day of DHA or placebo for over 6 months, indicated that larger doses of DHA are needed for adequate brain bioavailability, as confirmed by analysis of the cerebrospinal fluid (43). Moreover, a stratification of study participants by APOE4 status revealed its association with the level of delivery of DHA and EPA to the brain before the onset of cognitive impairment. These data, together with the DHA pharmacokinetics characteristics, should be carefully considered when setting up the DHA administration to patients at risk of cognitive impairment, as higher doses of DHA and longer periods of treatment could be necessary to obtain a meaningful remodeling of brain DHA levels. The relationship of plasma vitamin $\mathrm{E}$ and markers of vitamin $\mathrm{E}$ damage with $\mathrm{MCI}$ and $\mathrm{AD}$ has been investigated in cognitively normal subjects and in patients, revealing lower levels of tocopherols and vitamin $\mathrm{E}$ and increased vitamin $\mathrm{E}$ damage in $\mathrm{AD}$ and $\mathrm{MCI} v \boldsymbol{s}$. control subjects (44). However, vitamin E supplementation to $\mathrm{AD}$ patients produced contrasting results (45), suggesting the need for additional studies about this particular issue.

\section{DEPRESSION, BIPOLAR DISORDERS, OBSESSIVE-COMPULSIVE DISORDER: WHICH ROLE FOR NEUROINFLAMMATION?}

Neuropsychiatric disorders have been traditionally related to a dysregulation of neurotransmitters such as dopamine, norepinephrine, glutamate, and serotonin, contributing to their mood and cognitive dysfunction aspects. Nonetheless, the persistence of treatment-refractory conditions led to the hypothesis of a relationship between inflammation and neuropsychiatric disorders, supported by the observation that medical conditions associated with chronic inflammatory and immunological abnormalities, including obesity, diabetes, malignancies, rheumatoid arthritis, and multiple sclerosis, are risk factors for depression and bipolar disorder $(46,47)$. In addition, peripheral immune modulators, such as proinflammatory interleukin-1 $\beta$ (IL-1 $\beta$ ) and tumor necrosis factor$\alpha$ (TNF- $\alpha)$, can induce psychiatric symptoms in animal models (11). Finally, peripheral cellular and humoral immunological abnormalities are more prevalent in psychiatric $v s$. healthy control subjects (48-51). Of major interest, prospective studies revealed that high C-reactive protein (CRP) levels can predict a subsequent development of bipolar disorder, while increased levels of CRP or IL-6 are predictive of MDD and psychosis, thus suggesting a causal association between inflammatory status and neuropsychiatric disorders $(52,53)$.

MDD, bipolar disorders, and obsessive-compulsive disorder (OCD) are complex neuropsychiatric conditions with a set of causes and risks associated with gender, genetic and epigenetic factors, socioeconomic status, lifestyle, stress, alcohol and drug use, and concomitant disease. Some studies suggested a role also for unhealthy Western $\operatorname{diet}$ (54). BBB is a highly regulated interface that normally restricts the access to the brain of peripheral inflammatory mediators that could impair the neurotransmission. However, during peripheral inflammatory activation, the permeability of the BBB increases, and may exacerbate or initiate neuropsychiatric and neurological disorders (55). Neuroinflammation represents a reaction of the CNS to events that interfere with tissue homeostasis and is present in virtually all neurological diseases. Microglia function as innate immune cells of the CNS, playing key roles in clearing foreign particles and promoting brain healing after a trauma. Prolonged microglial activation, and in particular of the M1 pro-inflammatory microglia, can contribute to neuroinflammatory responses (56) also through endothelial and $\mathrm{BBB}$ dysfunction (57). Moreover, the oxidative stress caused by reactive oxygen/nitrogen species and mitochondrial disarrangements play key roles in neuroinflammation $(58,59)$. Pro-inflammatory cytokines and high nitric oxide (NO) levels produced by activated microglia may promote reactive oxygen species (ROS) formation (60). Due to its composition and biochemical characteristics, the brain is particularly vulnerable to oxidative stress, able to induce brain lipid peroxidation, damage to membrane phospholipids and to neurotransmitter receptors, and depletion of endogenous antioxidants (61). 
A strong relationship between the cytokine system and the neurotransmitter system has been highlighted. A special role in the pathogenesis and somatic consequences of MDD has been described for pro-inflammatory IL-6, whose activity may cause depression through the activation of the hypothalamic-pituitaryadrenal axis or influence of the neurotransmitter metabolism, as summarized elsewhere (62). Within the brain, the immunerelated information activates several regions and induces glia cells and neurons to release the same cytokines, in turn acting as neuro-regulators and neurotransmitters (63). In more detail, the link between inflammation and depression is probably based on the glucocorticoid insensitivity caused by inflammation, as well as on the deviation of the essential amino acid tryptophan from the production of serotonin toward the production of kynurenine and of its metabolites (e.g., quinolinic acid and kynurenic acid). The decreased synthesis of serotonin following the tryptophan shunting leads to an increased glutamatergic neurotransmission, known to be associated with a depressed mood (64).

\section{DEPRESSION, BIPOLAR DISORDERS, OBSESSIVE-COMPULSIVE DISORDER: AN ANTI-INFLAMMATORY TREATMENT APPROACH}

By virtue of the relationship between inflammation and neuropsychiatric disorders, increasing attention has been devoted to the therapeutic potential of anti-inflammatory treatments in this setting. Traditional antidepressants can have anti-inflammatory and neuroprotective effects, which might be partly due to their influence on cytokine production, as supported also by the decrease of circulating pro-inflammatory factors $(60,65)$. However, traditional antidepressants can induce side effects, in particular in case of premature selfdiscontinuation, and can be ineffective in $30-40 \%$ of patients. The enzymes cyclooxygenase- 1 (COX-1) and cyclooxygenase- 2 (COX-2) are involved in the inflammatory cascade leading to the production of prostacyclines, tromboxanes, and leukotrienes. An increasing number of studies are demonstrating that treatments targeting COX-1 and COX-2 can have a beneficial effect in the subgroup of depressed patients with elevated levels of pro-inflammatory cytokines and in patients affected by other neuropsychiatric disorders $(65,66)$. In particular, clinical studies concerning the use of non-steroidal anti-inflammatory drugs (NSAIDs) and depression revealed a beneficial effect in some cases, in particular in presence of inflammatory disease comorbidities that, however, has not been confirmed by other studies and needs additional investigation (65).

In addition to drug treatments, lifestyle changes, such as dietary interventions and/or the supplementation of specific micro- and macronutrients could have a beneficial effect on neuropsychiatric disorders, through an action on the immune system (67). Below we summarized the current evidences of the anti-inflammatory activity played by DHA, EPA, magnesium, alpha-tocopherol, and folate in the context of neuroinflammation.

\section{Focus on EPA, DHA and Neuroinflammation}

EPA and DHA have a well-known effect on synthesis, release, receptor function and storage of neurotransmitters during development and in neuropsychiatric disorders (68-71). In reference to inflammatory reactions, generally, DHA, and EPA exert an inhibitory effect on the activation of immune cells from both the innate and the adaptive systems. In particular, omega-3 PUFAs are able to act as signaling molecules and decrease cytokine secretion by macrophages and to suppress inflammasome-mediated inflammation. DHA and EPA are also able to decrease neutrophil migration and increase their phagocytic capacity (72). Of note, during the increase in vascular wall permeability, plasma omega-3 PUFAs reach the site of inflammation, where they are converted to SPM, able to block chronic inflammation and reduce fibrosis (73). A dietary approach including DHA, EPA, folic acid, and vitamin E exhibited beneficial effects on structural and functional recovery in a mouse model of transient middle cerebral artery occlusion, leading to decreased neuroinflammation and improved motor function (74). A national survey conducted in United States indicated a significant association between an EPA + DHA intake in the past $24 \mathrm{~h}$ and a reduced prevalence of depressive symptoms by $25 \%$ in a sample of 10,480 adults (75). A RCT conducted by Rapaport et al. (28) demonstrated a positive effect of EPA in a subset of subjects affected by MDD and with a high level of inflammatory biomarkers. A RCT conducted in depressed pregnant women revealed that the supplementation with EPA was associated with increased levels of estradiol during pregnancy and together they alleviated the antenatal depression. However, this process was presumably mediated by a mechanism other than anti-inflammation, as the relationship between depressive symptoms and inflammatory cytokines was not significant (29) (Table 1).

\section{Focus on Vitamin E and Neuroinflammation}

Alpha-tocopherol supplementation could modulate oxidative stress and inflammation in Parkinson's disease (PD) or AD, where neuroinflammation plays a major role. However, clear data from RCTs are still missing and indications are still limited mainly to preclinical research. The role of alphatocopherol in the brain has been investigated in a murine model with a double-deficiency of vitamins $\mathrm{C}$ and $\mathrm{E}$, revealing an increased expression of inflammatory-related genes, possibly causing neuroinflammation in the brain (76). In vitro and preclinical in vivo studies in a murine model of $\mathrm{AD}$ showed that the supplementation of alpha-tocopherol further decreased the neuroinflammation and oxidative stress induced by the candidate drug etodolac (77).

\section{Focus on Magnesium and Neuroinflammation}

Magnesium plays a key role in differentiation, proliferation, functioning, and movement of immune cells and is important for balanced immune cell responses $(15,78)$. Several studies evaluated serum levels of magnesium in different mental 
disorders; however, a pathophysiological association between mental disorders and magnesium in patients is not yet fully demonstrated. Some studies in murine models of disease revealed the positive role of magnesium in neuroinflammation reduction $(79,80)$. The large majority of studies carried out so far in patients have been focused on depression, because of the wellknown involvement of magnesium in several core mechanisms of depressive pathophysiology, including inflammation and oxidative stress (81). Among others, investigations proposed that magnesium could relieve depression by blocking the NMDA receptor, whose dysfunction is a major causative factor in depression pathology (82). Additional studies are required to establish the role of magnesium in pathophysiology or treatment of bipolar disorder and OCD.

\section{Focus on Folic Acid and Neuroinflammation}

Nearly $30 \%$ of severely depressed inpatients have folate deficiency, accompanied by a functional deficit reflected by increased plasma homocysteine (83). The rise in homocysteine indicates a failure of methylation of homocysteine to methionine, that, in turn, is a precursor of S-adenosylmethionine (SAM), the methyl donor in a number of brain reactions involving neurotransmitters, proteins, nucleoproteins, and membrane phospholipids. Similarly to folic acid, SAM has also been reported to affect mood (83-85). A similar correlation between folate, homocysteine, and disease status has been observed in patients affected by OCD (86). Of interest, hyperhomocysteinemia is associated with neuroinflammation in a murine model of vascular dementia (87). The use of folate-producing probiotic strains can be useful in reduction of neuroinflammation, as indicated by decreased levels of pro-inflammatory cytokines, and by improvement of motor behavior in PD (88). Some RCTs suggest that treatment with folate and vitamin B12 could be helpful in the long-term management of specific populations affected by depressive symptoms. However, the meta-analyses carried out by Almeida OP et al. (89) underlined the low number and heterogeneity of available studies, suggesting additional investigations.

\section{DISCUSSION}

Inadequate intake, systemic diseases, medical therapies, and genetic conditions can lead to deficiencies of specific nutrients, affecting both the central and peripheral nervous systems (3). A new research field, the "Nutritional Psychiatry," evolved in the last years with the aim to identify the dietary components that are particularly important for mental health, and, where appropriate, involve the prescription of dietary modification/supplementation to prevent or manage disorders (90). In addition, a personalized medicine approach has been proposed in the field of nutrition applied to mental well-being, based on the assessment of specific biomarkers (e.g., nutrient deficiencies, inflammatory cytokine levels, genomic assessment, microbiome analysis) to determine the individual macro- and micronutrient requirements (90). Overall, the research data we reviewed seem to support a positive role for the nutrients EPA,
DHA, magnesium, alpha-tocopherol, and folic acid, either alone or in combination with drugs, in the preservation of normal brain function and mental well-being, at least in part through the control of neuroinflammation (Figure 1). Additional clinical trials are warranted to confirm the results obtained so far and gain further insights on the mechanisms of actions and effective dose range of the nutrients EPA, DHA, magnesium, alpha-tocopherol, and folic acid on recovery/preservation of mental health and well-being. In particular, the studies we reviewed suggest that alpha-tocopherol supplementation is not consistently associated with improvements of symptoms of mental disorders. Considering the well-known physiological role played by this nutrient and its lower levels associated with mental disorders (91), we suggest that future adequately powered and properly designed clinical trials should further address this point.

The RCTs we reviewed included subjects of different age (Table 1). An adjustment of nutrients' intake and dosing is necessary in function of age range and of the associated assimilation and metabolism, and of the subjects' health status, with particular reference to elderly people, characterized by changes in physiology, metabolism, and organ function and where presence of comorbidities and/or the accumulation of particular nutrients (e.g., essential metals, as iron, copper, manganese) can contribute to the development of neurodegenerative diseases (92). This aspect should be considered when evaluating the results of RCTs and in the design of future studies focusing on the association between nutrients intake and preservation of mental health.

In addition to the published studies we reviewed, other clinical trials are currently on the way in the setting of nutrients and mental disorders or impairment. Several ongoing trials are focusing in particular on PUFAs: among them, the trial NCT03926351 is investigating the role of high-dose DHA in patients at risk for dementia, while the trial NCT03613844 is further investigating the association between the presence of the APOE $\varepsilon 4$ allele and the and the reduced delivery of DHA to the brain. Finally, LO MAPT is a phase III trial currently evaluating the efficacy of omega-3 supplementation on cognitive decline in older adults with low DHA/EPA status and subjective memory complaints or family history of Alzheimer disease (ClinicalTrials.gov Identifier: NCT03691519).

An accurate diagnosis of mental disorders and of their stage, alongside a detailed clinical history is of major importance in clinical trials testing the effectiveness of micro- and macronutrients, in order to enroll more homogeneous cohorts of patients, in turn leading to more reliable study results. Moreover, future research would greatly benefit from the use of more uniform tools to measure both functioning and cognition in patients affected by mental disorders. Finally, a nutritional approach based on multiple agents is recommendable rather than administration of single agent supplements.

\section{CONCLUSION}

Mental health is an integral and essential component of human health, and an unhealthy lifestyle can be associated with a poor 
mental health. Scientific findings encourage the integration of micro- and macronutrients in a well-balanced and varied diet, accompanied by a healthy lifestyle, for preservation of normal brain function and well-being. This strategy is of particular importance when considering the global human aging and that the brain suffers significantly from the life-long impact of stress factors.

\section{AUTHOR CONTRIBUTIONS}

MM drafted the manuscript and approved the submitted version.

\section{REFERENCES}

1. Marx W, Moseley G, Berk M, Jacka F. Nutritional psychiatry: the present state of the evidence. Proc Nutr Soc. (2017) 76:42736. doi: 10.1017/S0029665117002026

2. Adan RAH, van der Beek EM, Buitelaar JK, Cryan JF, Hebebrand J, Higgs S, et al. Nutritional psychiatry: towards improving mental health by what you eat. Eur Neuropsychopharmacol. (2019) 29:132132. doi: 10.1016/j.euroneuro.2019.10.011

3. Polavarapu A, Hasbani D. Neurological complications of nutritional disease. Semin Pediatr Neurol. (2017) 24:70-80. doi: 10.1016/j.spen.2016.12.002

4. Dyall SC. Long-chain omega-3 fatty acids and the brain: a review of the independent and shared effects of EPA, DPA and DHA. Front Aging Neurosci. (2015) 7:52. doi: 10.3389/fnagi.2015.00052

5. Brenna JT. Efficiency of conversion of alpha-linolenic acid to long chain n-3 fatty acids in man. Curr Opin Clin Nutr Metab Care. (2002) 5:12732. doi: 10.1097/00075197-200203000-00002

6. Bazinet RP, Metherel AH, Chen CT, Shaikh SR, Nadjar A, Joffre C, et al. Brain eicosapentaenoic acid metabolism as a lead for novel therapeutics in major depression. Brain Behav Immun. (2020) 85:2128. doi: 10.1016/j.bbi.2019.07.001

7. Denis I, Potier B, Vancassel S, Heberden C, Lavialle M. Omega-3 fatty acids and brain resistance to ageing and stress: body of evidence and possible mechanisms. Ageing Res Rev. (2013) 12:579-94. doi: 10.1016/j.arr.2013.01.007

8. Yang B, Lin L, Bazinet RP, Chien YC, Chang JP, Satyanarayanan SK, et al. clinical efficacy and biological regulations of $\omega$-3 PUFA-derived endocannabinoids in major depressive disorder. Psychother Psychosom. (2019) 88:215-24. doi: 10.1159/000501158

9. Rizvi S, Raza ST, Ahmed F, Ahmad A, Abbas S, Mahdi F. The role of vitamin E in human health and some diseases. Sultan Qaboos Univ Med J. (2014) 14:e157-65

10. Lewis ED, Meydani SN, Wu D. Regulatory role of vitamin $\mathrm{E}$ in the immune system and inflammation. IUBMB Life. (2019) 71:48794. doi: 10.1002/iub.1976

11. Traber MG. Vitamin E inadequacy in humans: causes and consequences. $A d v$ Nutr. (2014) 5:503-14. doi: 10.3945/an.114.006254

12. Leahy LG. Vitamin B Supplementation: What's the right choice for your patients? J Psychosoc Nurs Ment Health Serv. (2017) 55:7-11. doi: 10.3928/02793695-20170619-02

13. Elsherbiny NM, Sharma I, Kira D, Alhusban S, Samra YA, Jadeja R, et al. Homocysteine Induces Inflammation in Retina and Brain. Biomolecules. (2020) 10:393. doi: 10.3390/biom10030393

14. Nielsen FH. Magnesium deficiency and increased inflammation: current perspectives. J Inflamm Res. (2018) 11:25-34. doi: 10.2147/JIR.S136742

15. de Baaij JH, Hoenderop JG, Bindels RJ. Magnesium in man: implications for health and disease. Physiol Rev. (2015) 95:146. doi: 10.1152/physrev.00012.2014

16. Gonzalez MJ, Miranda-Massari JR. Diet and stress. Psychiatr Clin North Am. (2014) 37:579-89. doi: 10.1016/j.psc.2014.08.004

17. Kiecolt-Glaser JK. Stress, food, and inflammation: psychoneuroimmunology and nutrition at the cutting edge. Psychosom Med. (2010) 72:3659. doi: 10.1097/PSY.0b013e3181dbf489

\section{FUNDING}

Editorial assistance was supported by an unconditioned grant from Angelini Pharma S.p.A., Rome, Italy.

\section{ACKNOWLEDGMENTS}

Medical writing support was provided by Amalia Forte, $\mathrm{PhD}$, on behalf of Menthalia S.r.l., Naples, Italy.

18. Morey JN, Boggero IA, Scott AB, Segerstrom SC. Current directions in stress and human immune function. Curr Opin Psychol. (2015) 5:1317. doi: 10.1016/j.copsyc.2015.03.007

19. Oliver-Baxter JM, Whitford HS, Turnbull DA, Bond MJ. Effects of vitamin supplementation on inflammatory markers and psychological wellbeing among distressed women: a randomized controlled trial. J Integr Med. (2018) 16:322-28. doi: 10.1016/j.joim.2018.06.001

20. Pouteau E, Kabir-Ahmadi M, Noah L, Mazur A, Dye L, Hellhammer J, et al. Superiority of magnesium and vitamin B6 over magnesium alone on severe stress in healthy adults with low magnesemia: a randomized, single-blind clinical trial. PLoS ONE. (2018) 13:e0208454. doi: 10.1371/journal.pone.0208454

21. Bradbury J, Myers SP, Oliver C. An adaptogenic role for omega-3 fatty acids in stress; a randomised placebo controlled double blind intervention study (pilot) [ISRCTN22569553]. Nutr J. (2004) 3:20. doi: 10.1186/1475-2891-3-20

22. Jahangard L, Hedayati M, Abbasalipourkabir R, Haghighi M, Ahmadpanah M, Faryadras M, et al. Omega-3-polyunsatured fatty acids (O3PUFAs), compared to placebo, reduced symptoms of occupational burnout and lowered morning cortisol secretion. Psychoneuroendocrinology. (2019) 109:104384. doi: 10.1016/j.psyneuen.2019.104384

23. Amminger GP, Nelson B, Markulev C, Yuen HP, Schäfer MR, Berger M, et al. The NEURAPRO biomarker analysis: long-chain omega-3 fatty acids improve 6-month and 12-month outcomes in youths at ultra-high risk for psychosis. Biol Psychiatry. (2020) 87:43-252. doi: 10.1016/j.biopsych.2019.08.030

24. Robinson DG, Gallego JA, John M, Hanna LA, Zhang JP, Birnbaum $\mathrm{ML}$, et al. A potential role for adjunctive omega-3 polyunsaturated fatty acids for depression and anxiety symptoms in recent onset psychosis: results from a 16 week randomized placebo-controlled trial for participants concurrently treated with risperidone. Schizophr Res. (2019) 204:295303. doi: 10.1016/j.schres.2018.09.006

25. Jahangard L, Sadeghi A, Ahmadpanah M, Holsboer-Trachsler E, Sadeghi Bahmani D, Haghighi M, et al. Influence of adjuvant omega-3polyunsaturated fatty acids on depression, sleep, and emotion regulation among outpatients with major depressive disorders-Results from a doubleblind, randomized and placebo-controlled clinical trial. J Psychiatr Res. (2018) 107:48-56. doi: 10.1016/j.jpsychires.2018.09.016

26. Judge MP, Cong X, Harel O, Courville AB, Lammi-Keefe CJ. Maternal consumption of a DHA-containing functional food benefits infant sleep patterning: an early neurodevelopmental measure. Early Hum Dev. (2012) 88:531-7. doi: 10.1016/j.earlhumdev.2011.12.016

27. Stavrinou PS, Andreou E, Aphamis G, Pantzaris M, Ioannou M, Patrikios IS, et al. The effects of a 6-month high dose omega-3 and omega-6 polyunsaturated fatty acids and antioxidant vitamins supplementation on cognitive function and functional capacity in older adults with mild cognitive impairment. Nutrients. (2020) 12:325. doi: 10.3390/nu12020325

28. Rapaport MH, Nierenberg AA, Schettler PJ, Kinkead B, Cardoos A, Walker $\mathrm{R}$, et al. Inflammation as a predictive biomarker for response to omega- 3 fatty acids in major depressive disorder: a proof-of-concept study. Mol Psychiatry. (2016) 21:71-9. doi: 10.1038/mp.2015.22

29. Nishi D, Su KP, Usuda K, Chang JP, Hamazaki K, Ishima T, et al. Plasma estradiol levels and antidepressant effects of omega- 3 fatty acids in pregnant women. Brain Behav Immun. (2020) 85:29-34. doi: 10.1016/j.bbi.2019.02.014 
30. Poleszak E. Benzodiazepine/GABA(A) receptors are involved in magnesiuminduced anxiolytic-like behavior in mice. Pharmacol Rep. (2008) 60:483-9

31. Młyniec K, Gaweł M, Doboszewska U, Starowicz G, Nowak G. The role of elements in anxiety. Vitam Horm. (2017) 103:295-326. doi: 10.1016/bs.vh.2016.09.002

32. Boyle NB, Lawton C, Dye L. The effects of magnesium supplementation on subjective anxiety and stress-A systematic review. Nutrients. (2017) 9:429. doi: 10.3390/nu9050429

33. Desrumaux CM, Mansuy M, Lemaire S, Przybilski J, Le Guern N, Givalois $\mathrm{L}$, et al. Brain vitamin $\mathrm{E}$ deficiency during development is associated with increased glutamate levels and anxiety in adult mice. Front Behav Neurosci. (2018) 12:310. doi: 10.3389/fnbeh.2018.00310

34. Tanaka E, Yatsuya H, Uemura M, Murata C, Otsuka R, Toyoshima H, et al. Associations of protein, fat, and carbohydrate intakes with insomnia symptoms among middle-aged Japanese workers. J Epidemiol. (2013) 23:1328. doi: 10.2188/jea.JE20120101

35. Decoeur F, Benmamar-Badel A, Leyrolle Q, Persillet M, Layé S, Nadjar A. Dietary N-3 PUFA deficiency affects sleep-wake activity in basal condition and in response to an inflammatory challenge in mice. Brain Behav Immun. (2020) 85:162-9. doi: 10.1016/j.bbi.2019.05.016

36. Sanford AM. Mild cognitive impairment. Clin Geriatr Med. (2017) 33:32537. doi: 10.1016/j.cger.2017.02.005

37. Muscat SM, Barrientos RM. Lifestyle modifications with antineuroinflammatory benefits in the aging population. Exp Gerontol. (2020) 142:111144. doi: 10.1016/j.exger.2020.111144

38. de Waal H, Stam CJ, Lansbergen MM, Wieggers RL, Kamphuis PJ, Scheltens $\mathrm{P}$, et al. The effect of souvenaid on functional brain network organisation in patients with mild Alzheimer's disease: a randomised controlled study. PLoS ONE. (2014) 9:e86558. doi: 10.1371/journal.pone.0086558

39. Thaipisuttikul P, Galvin JE. Use of medical foods and nutritional approaches in the treatment of Alzheimer's disease. Clin Pract (Lond). (2012) 9:199209. doi: $10.2217 /$ cpr. 12.3

40. Onakpoya IJ, Heneghan CJ. The efficacy of supplementation with the novel medical food, Souvenaid, in patients with Alzheimer's disease: a systematic review and meta-analysis of randomized clinical trials. Nutr Neurosci. (2017) 20:219-27. doi: 10.1080/1028415X.2015.1110899

41. Thomas A, Baillet M, Proust-Lima C, Féart C, Foubert-Samier A, Helmer C, et al. Blood polyunsaturated omega-3 fatty acids, brain atrophy, cognitive decline, and dementia risk. Alzheimers Dement. (2020). doi: 10.1002/alz.12195. [Epub ahead of print].

42. Sydenham E, Dangour AD, Lim WS. Omega 3 fatty acid for the prevention of cognitive decline and dementia. Cochrane Database Syst Rev. (2012) 6:CD005379. doi: 10.1002/14651858.CD005379.pub3

43. Arellanes IC, Choe N, Solomon V, He X, Kavin B, Martinez AE, et al. Brain delivery of supplemental docosahexaenoic acid (DHA): a randomized placebo-controlled clinical trial. EBioMedicine. (2020) 59:102883. doi: 10.1016/j.ebiom.2020.102883

44. Mangialasche F, Xu W, Kivipelto M, Costanzi E, Ercolani S, Pigliautile $\mathrm{M}$, et al. Tocopherols and tocotrienols plasma levels are associated with cognitive impairment. Neurobiol Aging. (2012) 33:2282-90. doi: 10.1016/j.neurobiolaging.2011.11.019

45. Lloret A, Esteve D, Monllor P, Cervera-Ferri A, Lloret A. The effectiveness of vitamin $\mathrm{E}$ treatment in Alzheimer's disease. Int J Mol Sci. (2019) 20:879. doi: 10.3390/ijms20040879

46. Leboyer M, Soreca I, Scott J, Frye M, Henry C, Tamouza R, et al. Can bipolar disorder be viewed as a multi-system inflammatory disease? J Affect Disord. (2012) 141:1-10. doi: 10.1016/j.jad.2011.12.049

47. Dantzer R, O'Connor JC, Freund GG, Johnson RW, Kelley KW. From inflammation to sickness and depression: when the immune system subjugates the brain. Nat Rev Neurosci. (2008) 9:46-56. doi: 10.1038/nrn2297

48. Papakostas GI, Shelton RC, Kinrys G, Henry ME, Bakow BR, Lipkin SH, et al. Assessment of a multi-assay, serum-based biological diagnostic test for major depressive disorder: a pilot and replication study. Mol Psychiatry. (2013) 18:332-9. doi: $10.1038 / \mathrm{mp} .2011 .166$

49. Liu Y, Ho RC, Mak A. Interleukin (IL)-6, tumour necrosis factor alpha $(\mathrm{TNF}-\alpha)$ and soluble interleukin-2 receptors (sIL-2R) are elevated in patients with major depressive disorder: a meta-analysis and meta-regression. $J$ Affect Disord. (2012) 139:230-9. doi: 10.1016/j.jad.2011.08.003
50. Brietzke E, Stabellini R, Grassi-Oliveira R, Lafer B. Cytokines in bipolar disorder: recent findings, deleterious effects but promise for future therapeutics. CNS Spectr. (2011) 16:157-68. doi: 10.1017/S1092852912000338 51. Turna J, Grosman Kaplan K, Anglin R, Patterson B, Soreni N, Bercik $\mathrm{P}$, et al. The gut microbiome and inflammation in obsessive-compulsive disorder patients compared to age- and sex-matched controls: a pilot study. Acta Psychiatr Scand. (2020) 142:337-47. doi: 10.1111/acps. 13175

52. Wium-Andersen MK, Ørsted DD, Nordestgaard BG. Elevated C-reactive protein and late-onset bipolar disorder in $78 \quad 809$ individuals from the general population. $\mathrm{Br} J$ Psychiatry. (2016) 208:138-45. doi: 10.1192/bjp.bp.114.150870

53. Khandaker GM, Pearson RM, Zammit S, Lewis G, Jones PB. Association of serum interleukin 6 and C-reactive protein in childhood with depression and psychosis in young adult life: a population-based longitudinal study. JAMA Psychiatry. (2014) 71:1121-8. doi: 10.1001/jamapsychiatry.2014.1332

54. Kris-Etherton PM, Petersen KS, Hibbeln JR, Hurley D, Kolick V, Peoples S, et al. Nutrition and behavioral health disorders: depression and anxiety. Nutr Rev. (2020) 24:nuaa025. doi: 10.1093/nutrit/nuaa025

55. Morris G, Fernandes BS, Puri BK, Walker AJ, Carvalho AF, Berk M. Leaky brain in neurological and psychiatric disorders: drivers and consequences. Aust N Z J Psychiatry. (2018) 52:924-48. doi: 10.1177/0004867418796955

56. DiSabato DJ, Quan N, Godbout JP. Neuroinflammation: the devil is in the details. J Neurochem. (2016) 139(Suppl.2):136-53. doi: 10.1111/jnc.13607

57. Ronaldson PT, Davis TP. Regulation of blood-brain barrier integrity by microglia in health and disease: a therapeutic opportunity. J Cereb Blood Flow Metab. (2020) 14:271678X20951995. doi: 10.1177/0271678X20951995

58. Picca A, Calvani R, Coelho-Junior HJ, Landi F, Bernabei R, Marzetti E. Mitochondrial dysfunction, oxidative stress, and neuroinflammation: intertwined roads to neurodegeneration. Antioxidants (Basel). (2020) 9:647. doi: 10.3390/antiox9080647

59. Khadka B, Lee JY, Park DH, Kim KT, Bae JS. The role of natural compounds and their nanocarriers in the treatment of CNS inflammation. Biomolecules. (2020) 10:E1401. doi: 10.3390/biom10101401

60. Jia X, Gao Z, Hu H. Microglia in depression: current perspectives. Sci China Life Sci. (2020). doi: 10.1007/s11427-020-1815-6. [Epub ahead of print].

61. Najjar S, Pearlman DM, Alper K, Najjar A, Devinsky O. Neuroinflammation and psychiatric illness. J Neuroinflammation. (2013) 10:43. doi: 10.1186/1742-2094-10-43

62. Ting EY, Yang AC, Tsai SJ. Role of interleukin-6 in depressive disorder. Int $J$ Mol Sci. (2020) 21:2194. doi: 10.3390/ijms21062194

63. Pollak Y, Yirmiya R. Cytokine-induced changes in mood and behaviour: implications for 'depression due to a general medical condition', immunotherapy and antidepressive treatment. Int J Neuropsychopharmacol. (2002) 5:389-99. doi: 10.1017/S1461145702003152

64. Müller N, Schwarz MJ. The immune-mediated alteration of serotonin and glutamate: towards an integrated view of depression. Mol Psychiatry. (2007) 12:988-1000. doi: 10.1038/sj.mp.4002006

65. Kopschina Feltes P, Doorduin J, Klein HC, Juárez-Orozco LE, Dierckx RA, Moriguchi-Jeckel CM, et al. Anti-inflammatory treatment for major depressive disorder: implications for patients with an elevated immune profile and non-responders to standard antidepressant therapy. J Psychopharmacol. (2017) 31:1149-65. doi: 10.1177/0269881117 711708

66. Sethi R, Gómez-Coronado N, Walker AJ, Robertson OD, Agustini B, Berk M, et al. Neurobiology and therapeutic potential of cyclooxygenase-2 (COX-2) inhibitors for inflammation in neuropsychiatric disorders. Front Psychiatry. (2019) 10:605. doi: 10.3389/fpsyt.2019.00605

67. Gombart AF, Pierre A, Maggini S. A review of micronutrients and the immune system-working in harmony to reduce the risk of infection. Nutrients. (2020) 12:236. doi: $10.3390 /$ nu12010236

68. Patrick RP, Ames BN. Vitamin D and the omega-3 fatty acids control serotonin synthesis and action, part 2: relevance for ADHD, bipolar disorder, schizophrenia, and impulsive behavior. FASEB J. (2015) 29:220722. doi: $10.1096 / f$ j. $14-268342$

69. Su KP. Biological mechanism of antidepressant effect of omega- 3 fatty acids: how does fish oil act as a 'mind-body interface'? Neurosignals. (2009) 17:14452. doi: $10.1159 / 000198167$ 
70. Molfino A, Amabile MI, Monti M, Muscaritoli M. Omega-3 polyunsaturated fatty acids in critical illness: anti-inflammatory, proresolving, or both? Oxid Med Cell Longev. (2017) 2017:5987082. doi: 10.1155/2017/5987082

71. Molfino A, Gioia G, Rossi Fanelli F, Muscaritoli M. The role for dietary omega3 fatty acids supplementation in older adults. Nutrients. (2014) 6:405873. doi: $10.3390 /$ nu6104058

72. Gutiérrez S, Svahn SL, Johansson ME. Effects of omega-3 fatty acids on immune cells. Int J Mol Sci. (2019) 20:5028. doi: 10.3390/ijms20205028

73. Parolini C. Marine $n-3$ polyunsaturated fatty acids: efficacy on inflammatorybased disorders. Life Sci. (2020) 15:118591. doi: 10.1016/j.lfs.2020.118591

74. Wiesmann M, Zinnhardt B, Reinhardt D, Eligehausen S, Wachsmuth L, Hermann S, et al. A specific dietary intervention to restore brain structure and function after ischemic stroke. Theranostics. (2017) 7:493512. doi: 10.7150/thno.17559

75. Hoffmire CA, Block RC, Thevenet-Morrison K, van Wijngaarden E. Associations between omega- 3 poly-unsaturated fatty acids from fish consumption and severity of depressive symptoms: an analysis of the 20052008 National Health and Nutrition Examination Survey. Prostaglandins Leukot Essent Fatty Acids. (2012) 86:155-60. doi: 10.1016/j.plefa.2012.03.003

76. Takahashi K, Yanai S, Takisawa S, Kono N, Arai H, Nishida Y, et al. Vitamin C and vitamin E double-deficiency increased neuroinflammation and impaired conditioned fear memory. Arch Biochem Biophys. (2019) 663:1208. doi: 10.1016/j.abb.2019.01.003

77. Elfakhri KH, Abdallah IM, Brannen AD, Kaddoumi A. Multi-faceted therapeutic strategy for treatment of Alzheimer's disease by concurrent administration of etodolac and $\alpha$-tocopherol. Neurobiol Dis. (2019) 125:12334. doi: 10.1016/j.nbd.2019.01.020

78. Brandao K, Deason-Towne F, Perraud AL, Schmitz C. The role of $\mathrm{Mg} 2+$ in immune cells. Immunol Res. (2013) 55:261-9. doi: 10.1007/s12026-012-8371-X

79. Johnson AC, Tremble SM, Chan SL, Moseley J, LaMarca B, Nagle KJ, et al. Magnesium sulfate treatment reverses seizure susceptibility and decreases neuroinflammation in a rat model of severe preeclampsia. PLoS ONE. (2014) 9:e113670. doi: 10.1371/journal.pone.0113670

80. Wang P, Yu X, Guan PP, Guo JW, Wang Y, Zhang Y, et al. Magnesium ion influx reduces neuroinflammation in $\mathrm{A} \beta$ precursor protein/Presenilin 1 transgenic mice by suppressing the expression of interleukin-1 $\beta$. Cell $\mathrm{Mol}$ Immunol. (2017) 14:451-64. doi: 10.1038/cmi.2015.93

81. Botturi A, Ciappolino V, Delvecchio G, Boscutti A, Viscardi B, Brambilla $P$. The role and the effect of magnesium in mental disorders: a systematic review. Nutrients. (2020) 12:1661. doi: 10.3390/nu12 061661

82. Pittenger C, Sanacora G, Krystal JH. The NMDA receptor as a therapeutic target in major depressive disorder. CNS Neurol Disord Drug Targets. (2007) 6:101-15. doi: $10.2174 / 187152707780363267$
83. Bottiglieri $\mathrm{T}$, Laundy $\mathrm{M}$, Crellin $\mathrm{R}$, Toone BK, Carney MW, Reynolds EH. Homocysteine, folate, methylation, and monoamine metabolism in depression. J Neurol Neurosurg Psychiatry. (2000) 69:228-32. doi: 10.1136/jnnp.69.2.228

84. Bottiglieri T, Hyland K, Reynolds EH. The clinical potential of ademetionine (S-adenosylmethionine) in neurological disorders. Drugs. (1994) 48:13752. doi: 10.2165/00003495-199448020-00002

85. Bender A, Hagan KE, Kingston N. The association of folate and depression: a meta-analysis. J Psychiatr Res. (2017) 95:918. doi: 10.1016/j.jpsychires.2017.07.019

86. Atmaca M, Tezcan E, Kuloglu M, Kirtas O, Ustundag B. Serum folate and homocysteine levels in patients with obsessivecompulsive disorder. Psychiatry Clin Neurosci. (2005) 59:61620. doi: 10.1111/j.1440-1819.2005.01425.x

87. Sudduth TL, Powell DK, Smith CD, Greenstein A, Wilcock DM. Induction of hyperhomocysteinemia models vascular dementia by induction of cerebral microhemorrhages and neuroinflammation. J Cereb Blood Flow Metab. (2013) 33:708-15. doi: 10.1038/jcbfm.2013.1

88. Perez Visñuk D, Savoy de Giori G, LeBlanc JG, de Moreno de LeBlanc A. Neuroprotective effects associated with immune modulation by selected lactic acid bacteria in a Parkinson's disease model. Nutrition. (2020) 7980:110995. doi: 10.1016/j.nut.2020.110995

89. Almeida OP, Ford AH, Flicker L. Systematic review and meta-analysis of randomized placebo-controlled trials of folate and vitamin B12 for depression. Int Psychogeriatr. (2015) 27:727-37. doi: 10.1017/S1041610215000046

90. Sarris J. Nutritional psychiatry: from concept to the clinic. Drugs. (2019) 79:929-34. doi: 10.1007/s40265-019-01134-9

91. Casati M, Boccardi V, Ferri E, Bertagnoli L, Bastiani P, Ciccone S, et al. Vitamin $\mathrm{E}$ and Alzheimer's disease: the mediating role of cellular aging. Aging Clin Exp Res. (2020) 32:459-64. doi: 10.1007/s40520-019-01209-3

92. Mezzaroba L, Alfieri DF, Colado Simão AN, Vissoci Reiche EM. The role of zinc, copper, manganese and iron in neurodegenerative diseases. Neurotoxicology. (2019) 74:230-41. doi: 10.1016/j.neuro.2019.07.007

Conflict of Interest: The author declares that the research was conducted in the absence of any commercial or financial relationships that could be construed as a potential conflict of interest.

Copyright (ㄷ) 2021 Muscaritoli. This is an open-access article distributed under the terms of the Creative Commons Attribution License (CC BY). The use, distribution or reproduction in other forums is permitted, provided the original author(s) and the copyright owner(s) are credited and that the original publication in this journal is cited, in accordance with accepted academic practice. No use, distribution or reproduction is permitted which does not comply with these terms. 